Fesetzten Reagenzien ist von ausschlaggebender Bedeuting, es wurde deshalb stets die gleiche: $\mathrm{H}_{2} \mathrm{O}_{2}$, KM Mn $\mathrm{O}_{4}$ und Alkali gewält. Der Elek trolyt, dessen Einfluß studiert werden sollie, wurde dem Wasser zuerst zugesetzt.

2. Die Annabme, dab cias Ansteigen der Reaktionsgeschwind gikeitskonstanten auf Bildung und nachherigen Zerfall eires Wasserstoffperoxydsalzes durch den Verlauf der Reaktion zu erkiären sei, konnte nicht bestätigt werden.

3. Der Einflub der Kationen zugesetzten Salze mit gleichem Anion ordnet sich nach der lyotropen Reihe $\stackrel{+}{\mathrm{Ba}}>\mathrm{Sr}>\mathrm{Ca}>\mathrm{Na}>\mathrm{K}>\frac{+}{\mathrm{Li}}$, so da $\stackrel{+}{\mathrm{Ba}}$ die grölte Beschleunigung, $\stackrel{+}{\mathrm{Li}}$ die größte Hemmung auf die Katalyse ausübt. Bei böheren Konzentrationen tritt $\stackrel{+}{\mathrm{Ba}}$ binter $\stackrel{+}{\mathrm{Ca}}$

4. $\mathrm{Mg}$ und $\mathrm{NH}_{4}$ wirken durch starke Herabminderung der Hydroxylionenkonzentration ungeruein stark hemmend.

Sucht man die gefundenen Gesetzmäbigkeiten praktisch für Sauerstoffbäder zu ver- werten, so ist vor allen Dingen die Verwendung magnesiaharten Wassers zu vermeiden. Dagegen kan ein Gips oder noch besset Chlorkaluinm. zusatz durch Beschleunigung der Katalyse günstig wirken, und zwar auch in dem Falle, wenn ein Brunnenwasser infolge seiner Härte eine zu starke Hemmung der Katalyse herbeiführen sollte. Es wurde nämich durch besondere Versuche diese günstige Wirkung namentlich des Gipses lestgestellt.

Immerhin ist die Notwendigkeit gewöhnliches Gebrauchswasser für eine gegen Salz. einflüsse und atsch andere Einwirkungen, deren Ausschaltung man nicht in der Hand hat, so empfindliche Reaktion, wie es eine katalytische ist, anwenden $z u$ mủssen eine sehr mibliche Sache, da man höchstens dann eine richtige Dosierung der Reagenzien feststellen könnte, wenn man mit dem zur Verfügung stehenden Wasser einen Vorversuch angestellt hatte. Das läßt sich aber praktisch, da sich ein Laie damit befassen müßte, gar nicht durchführen.

Dresden.

Technische Hochschule.

\title{
Untersuchungen über die Viskosität von Azetylzeliulosen.
}

Von Anna von Fischer. (Bern-Zollikofen). (Eingegargen an 1. Juli 1221.) (Aus dem Laboratorium der Schweiz, Zelluloidfabrik, Bern-Zollikofen.)

Einleitung.

Die Azetylzellulose hat in den letzten Jahren in der Industrie eine ziemlich bedeutende Rolle gespielt. Sie wurde zur Herstellung von Plugzeuglacken, Kinofilms und zelluloidartigen Massen verwendet. Die letzteren wurden zur Kriegszeit in der Schweiz zu Gasmaskeng!äsern und Sturn" laternenscheiben verarbeitet. Trotz dieser mannigfaltigen Anwendungsgebiete blieb die Viskosität der Azetylzeliulose - ein bei kolloiden Substanzen so wichtiger Faktor - sozusagen unerforscht. Die einzige Arbeit, die ich dartiber in der fruheren Literatur finden konnte, ist die von Barth élem y '). Neben einer Beschreibung der gebräuchlichsten Viskosimeter befindet sich darin eine Zusammenstellung der Viskositätsuntersuchungen an Nitro-, Azetylzellulosen und
Zellulosexanthogenat. Die eigenen Versuche des Autors behandeln die Wirkung der Zeit, der Temperatur und verschiedener Lösungsmittel auf die Viskosität fünfprozentiger Azetylzelluloselösungen.

In den Fabriken, wo die Azetylzellulose hergestellt wurde, arbeitete man allerdings mit Viskositäten; doch begnügte mun sich da mit der Bestimmung eines einzigen Viskositätspunktes. Zur Charakterisierung der Azetylzellulose sollte die erhaltene sog. Viskositätszahl genügen. Man verstand darunter den Quotienten aus der Durchthußzeit der Azetylzelluloselösung und derjenigen der Vergleichsflüssigkeit.

Als Vergleichsflüssigheit diente entweder destilliertes Wasser (Barthe le my) oder reines Glyzerin.

$$
\text { Viskositätszahl }=\frac{\text { Durchflußzzeit d. A.-Z. Lösung }}{\text { Durchfiußzeit d. Vergleichsflüssigkeit }}
$$

In unserem Betrieb wurde die Azetylzellulose zur Darstellung eines unbrenbaren zellu-

i) H. Barthélemy, Le Caoutchouc et la Guttapercha $10,7202(1913)$. loidähnlichen Stoffes verwendet. Es wurde eine meiner Aufgaben in Laboratorium die Brauch. barkeit der einzeinen Azetylzellulosen für diese Fabrikation zu prüfen. Zu diesen Zweck wurde 
die Vistositatsahl sechprozoniger Azetylyelu. loselösungen in Azeron bestimt, wobei als Vergleichsflussigieit reines Glyzerin diente. Schon nacin whigen Versuchen wat ersichtlich, daß diese Methode keine richtigen Anbaitspunkte für den Betrieb zu geben yermochte. Es verhielten sich nämlich Azetyizelitosen von übereinstimmender Visirociasuahl bei der Fabrikation des zelluloidăhnichen Stoffes ganz verschieden, indem die einen sehr gute, die anderen unbrauchbare Fertigware lieferten. Die Viskositätszah: war also nicht imstande, eine Azety!zellulose zn charakterisieren.

Andererseits wurde beobachtet, daR die Viskosität der Azetylzelluloselösungen in Azeton durch Zusatz von Aethylalkohol, der aufi die Azetylzellulose als Fällungsmittel wirkt, beträchtlich beeinfluBt wird. Auf Grund dieser Tatsache unternahm ich es, eine neve Untersuchungsmethode autzubater. Anstatt eines einzigen Viskositätspunktes wurden die Viskositärs-Alkoholkonzentrationskurven siebenprozentiger Azetylzelluloselösunger in Azeton bestinmt ${ }^{2}$ ).

Arbeitsmethode.

Zunächst mubte nach einem passenden Viskosimeter gesucht werden. Zur Bestinmung der AusfluBgeschwindigkeit kann man eine gewöhnliche Pipette verwenden. Dies ist für eip Betriebslaboratorium die einfachste und zugänglichste Methode, doch ist sie nicht vollstăndig zuveriässig wegen der schwierigen Temperaturregulierung. Aus dem gleichen Grunde ist das Viskosineter von $\mathrm{Coch} \mathrm{iu} \mathrm{s}^{3}$ ) nicht gut anwendbar, das auzerdem die, manchmal sehr geringen Unterschiede nicht anzugeben vermag. Der Engler'sche Apparat 4) ist für Betriebszwecke uapraktisch, da er grobe Füssigkeitsmengen erfordert. Nach vielen miBiungenen Versucken erwies sich endich das Kapillarviskosimeter von Ostwald als gut brauchbar. Die Dimensionen des Apparates wurden in Anbetracht der hohen Viskositu der Lösungen folgendermaben modifiziert:

Inhal der Kugel $\quad=25 \mathrm{ccm}$

Durchmesser der Kapillare $=2,5 \mathrm{~mm}$

Lânge der Kapillare $=150 \mathrm{~mm}$

Zur Konstanthaltung der Temperatur wurde das Viskosimeter in ein hohes Becherglas mit

a) Einer kumlich erschienenen Arbeit von $\mathrm{Guy}$ Barr and 1. L. Bitcumshaw IKol1.-Zeitschr. 28 , 223) liegt der gleiche Gedanke za Grunde.

3) C. Piest, Ueber fie Viskositut von Nitrozellyloselosungen [Zeitschr. 1. angewandte Chemie 24, 968 $(1911)$.

4) H.Nishida, Kunststoffe 4,81 u. $105(1914 / 15)$.
Wasser gesteilt. Dic Versuchstemperatur wurde aut 18 Grad $C$ festgesetzt.

Als Lösungsmittel für die Azetylzellulosen diente reines Azeton und Gemische von Azeton und Aethylalkohol, welche 70,80 und 90 Vol.Proz. Azeton enthielten. Das Azeton war frisch destilliert und zeigte einen Siedepurikt von 56,5 Grad C. Der Aethylalkohol war 96 grädiger mit 0,5 Proz. Kampler denaturierter Handelsalkohol.

Die Azélylzelluloselösungen wurden folgendermabern hergestelli: In $100 \mathrm{ccm}$ des Lösungsmittels wurcen $7 \mathrm{~g}$ Azetylzellulose am Abend durch dreistündiges Schütteln in der Schüttelmaschine gelöst. Die Lösungen wurden über Nacht stehen gelassen und im Laufe des näch. sten Vornütags gemessen, wobei stets $50 \mathrm{ccm}$ der Lösung ins Viskosineter eingefült wurden. Durch das Steheniassen batten sich allfallige unlösliche Fasern und Gelklümpchen vollständig abgesetzt.

Von jeder Lösung wurden sechs Viskositäisbestimmungen gemacht und der Mittelwert berechnet. Dabei ergab sich bei Durchflufizeiten bis zu fünf Minuten ein Versuchsfehler von höchstens 0,5 Proz., bei Durchflufzeiten von fünf bis elf Minuten ein solcher von ein bis höchstens zwei Proz. Die Genauigkeit der Messungen war also sehr befriedigend.

\section{Versuchsergebnisse.}

Es wurden im Ganzen 39 Azetylzellulosen untersucht, die drei verschiedenen Fabriken entstammten. Die Versuche ergaben sieben typische Viskositätskurven, welche in der nachfolgenden Tabelle 1ud in Fig. 1 und 2 wiedergegeben sind.

Es stellte sich bei den Versuchen deutlich heraus, dab nicht die absolute Gröbe der Viskosität, sondern nur der Verlauf der Kurve für die Beurteilung der Azetylzellulose maßgebend ist.

Die in Fig. 1 wiedergegebenen Kurven und ihre Analoga waren charakteristisch für Azetylzellulosen, welche in Betrieb gute Resultate lieferten. Das Material war auf der Walze verhallnismäBig "lang". Es war plastisch und zeigte in Block nach dem Kochen gute Verwachsung. Die in Fig. 2 dargesteliten Kurven waren dagegen typisch für Azetylzellulosen, welche sich fïr die Fabrikation des zellinloidähnlichen Stoffes als gänzlich unbrauckbar erwiesen. Das Material war schon in der Mischmaschine "kurz", bröckelte auf der Walze auseinander, oder es zeigte sich im besten Fall nach dem Kochprozeb gar keine Bindung; ter Block hatte Risse und bläterte ab. 


\begin{tabular}{|c|c|c|c|c|c|c|c|}
\hline \multirow{2}{*}{$\begin{array}{l}\text { Azeton- } \\
\text { konzentration } \\
\text { Vol. Proz. }\end{array}$} & \multicolumn{7}{|c|}{ Viskosität } \\
\hline & Typ. 1 & 2 & 3 & 4 & 5 & 6 & 7 \\
\hline $\begin{array}{r}70 \\
80 \\
90 \\
100\end{array}$ & $\begin{array}{r}8^{\prime} 499^{\prime \prime} \\
7^{\prime} 44^{\prime \prime} \\
8^{\prime} \cdot 35 \% \\
13 \cdot 31^{\prime \prime}\end{array}$ & $\begin{array}{c}8.3 " \prime \\
7.33^{\prime \prime} \\
74^{\prime \prime \prime} \\
10.58 "\end{array}$ & $\begin{array}{c}8.9{ }^{\prime \prime} \\
7 \cdot 36^{\prime \prime} \\
8^{\circ} 46^{\prime \prime} \\
26^{\prime} 37^{\prime \prime}\end{array}$ & $\begin{array}{c}10^{\prime} 55^{\prime \prime} \\
7 \cdot 22^{\prime \prime} \\
6^{\prime} 3^{\prime \prime} \\
121^{\prime} 11^{\prime \prime}\end{array}$ & $\begin{array}{l}8^{\prime} 42^{\prime \prime} \\
7^{\prime} 14^{\prime \prime} \\
6^{\prime} 56^{\prime \prime} \\
8^{\prime} 51^{\prime \prime}\end{array}$ & $\begin{array}{l}5^{\prime} 25^{\prime \prime} \\
4^{\prime} 1^{\prime \prime} \\
3^{\prime} 30^{\prime \prime} \\
4^{\prime} 50^{\prime \prime}\end{array}$ & $\begin{array}{c}6.36^{\prime \prime} \\
6^{\prime} 1^{\prime \prime} \\
6^{\prime} 29^{\prime \prime} \\
11^{\prime} 37^{\prime \prime}\end{array}$ \\
\hline
\end{tabular}



Fig. 1



Fig.2
Figur 1. Bei näherer Betrachtung der Kurven in Fig. 1 ist ersichtlich, dab für alle guten Azetylzellulosen ein Viskositätsminimum in Alkoholazetonlösungen mit 80 Vol.- Proz. Azeton besteht. Die Azetylzellulosen des Typ. 2 zeichnen sich durch den fast unmerklichen Viskositätsunterschied bei Alkoholazetonlösungen mit 80 und 90 Vol.-Proz. Azeton aus. Typ. 3 weist einen enormen Viskositätsanstieg in reinem Azeton auf. Als Extrem dieser Art wurde eine Azetyizellulose gefunden, welche sich in reinem Azeton überhatipt nicht mehr völlig löste, sondern - nur zu einer dicken, durchscheinenden Gallerte aufquoll. Bei einigen Vertretern dieses Typus ver- schiebt sich das Viskositätsminimum auf die $\mathrm{Al}$ koholazetonlösung mit 70 Vol.-Proz. Azeton, z. B.

$$
\begin{array}{cc}
\begin{array}{c}
\text { Azetonkonzentration } \\
\text { Vol.Proz. }
\end{array} & \text { Viskosität } \\
70 & 9^{\prime} 58^{\prime \prime} \\
80 & 10^{\prime} 16^{\prime \prime} \\
90 & 11^{\prime} 36^{\prime \prime} \\
100 & 33^{\prime} 10^{\prime \prime}
\end{array}
$$

Bezeichnend für die guten Azetylzellulosen ist ferner die Tatsache, dab sie stets scharf ausgeprägte Kurvenbilder aufweisen,

Figur 2. Die Kurven in Fig. 2 sind charakteristisch für schlechte Azetylzellulosen. Die Kurvenbilder sind da nicht immer scharf ausgeprägt. So erhält man bei manchen schlechten Azetylzellulosen eine Kurve, welche ein Mittelding zwischen Typ. 4 und Typ. 5 darstellt tund bei der nur das charakteristische Merkmal des Viskositätsminimums in der Alkoholazetonlösung mit 90 Vol.- Proz. Azeton beibehalten bleibt, z. B:

$\begin{array}{cc}\begin{array}{c}\text { Azetonkonzentration } \\ \text { Vol.-Proz. }\end{array} & \text { Viskosität } \\ 70 & 3^{\prime} 7^{\prime \prime} \\ 80 & 2^{\prime} 53^{\prime \prime} \\ 90 & 2^{\prime} 43^{\prime \prime} \\ 100 & 4^{\prime} 2^{\prime \prime}\end{array}$

In ihrer Art gut ausgeprägt sind die Azetylzellulosen vom Typ. 6 mit ihrem Viskositätsmaximum in Alkoholazetonlösungen mit 70 Vol.Proz. Azeton. Ein Extrem dieser Art war eine Azetylzellulose, welche im Alkohol-Azetongenisch mit 70 Vol.- Proz. Azeton überhaupt nicht mehr löslich war, sondern nur aufquoll.

Eine Eigentümlichkeit zeigte sich bei Typ. 1 (Fig. 1) und Typ. 7 (Fig. 2). Die beiden Kurven sind in ihrem Verlauf sozusagen vollständig analog und doch lieferten die Azetylzellulosen vom Typ. 1 die besten, die vorn Typ. 7 die schlechtesten Betriebsresultate. In diesen beiden Typen vermag aliso auch die Viskositätskurve nicht die Azetylzellulosen zu charakterisieren. Trotz vieler und mannigfaltiger Versuche, bei verschiedener Temperatur und mit anderen $\mathrm{Zu}$ sätzen, gelang es mir leider nicht, dieses Rätsel zu lösen. 
Die 39 untersuchten Azetylzellulosen entstammten den nachstehenden drei Fabriken: Dreyfub in Basel, Usines du Rhône und Usines de Gr. Quevilly. Sie verteilten sich auf die sieben Typen, wie in der folgenden Tabelle dargestellt ist.

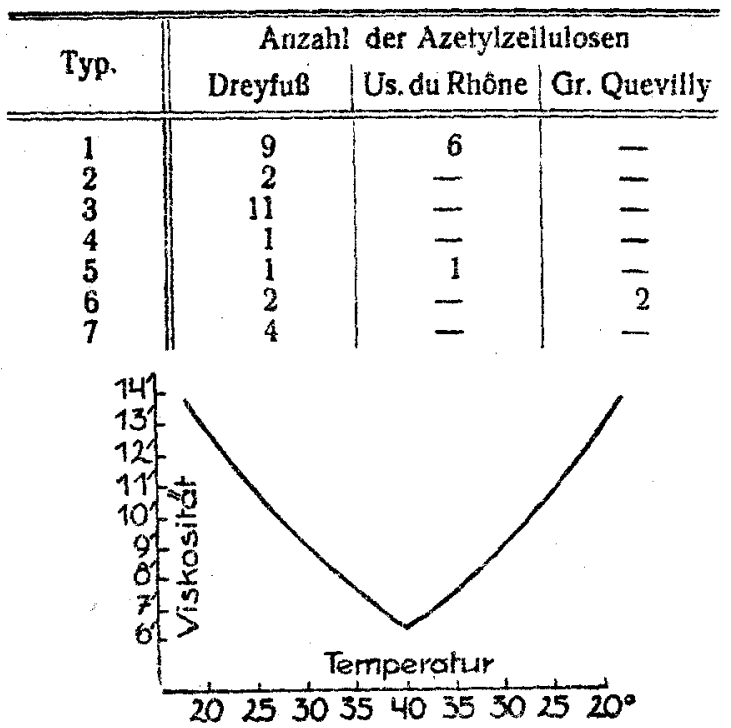

Fig. 3

Einflub der Temperatur.

Die Versuche wurden mit 7 prozentigen Azetylzelluloselösungen in reinem Azeton ausgeführt:. Die Lösung wurde im Viskosimeter langsam erwärmt und von fünf zu fünf Grad gemessen. Beim nachherigen allmählichen $\mathrm{Er}$ kalten wurde wiederum von fünf zu fünf Grad untersucht. Es ergab sich eine analoge Kurve, wie sie $\mathrm{Schwar} \mathrm{c}^{5}$ ) für Nitrozelluloselösungen in Kampferalkohol gefunden hat. Der Prozeb ist also auch bei Azetylzellulosen reversibel. (Siehe Fig. 3.)

Temp.: $\quad \begin{array}{lllll}18^{\circ} & 25^{\circ} & 30^{\circ} & 35^{\circ} & 40^{\circ}\end{array}$ Viskos.: $13^{\prime 4} 48^{\prime \prime} 10^{\prime} 41^{\prime \prime} 9^{\prime} 9^{\prime \prime} 7^{\prime 3} 31^{\prime \prime} 6^{\prime} 18^{\prime \prime}$

Temp.: $\quad 35^{\circ} \quad 30^{\circ} 25^{\circ} \quad 18^{\circ} \mathrm{C}$ Viskios.: 7“35" $9^{\prime} 12^{\prime \prime} 10^{\prime 4} 1^{\prime \prime} 13^{\prime} 48^{\prime \prime}$

Einflub von Zusätzen.

Der Einfluh von Zusätzen wurde an dreiprozentigen Azetylzelluloselösungen untersucht. Zur Messung diente ein Ostwald'sches Viskosimeter von folgenden Dimensionen:

lnhait der Kugel $=25 \mathrm{ccm}$

Durchmesser der Kapillare $=2 \mathrm{~mm}$

Länge der Kapillare $\quad=150 \mathrm{~mm}$

5) H. Schwarz, Koll.-Zeitschr. 12, 32 (1913).
Die Versuchstemperatur betrug $18 \mathrm{Grad} \mathrm{C}$. Auch da wurden die Lösungen am Ábend angesetzt, drei Stunden lang in der Schüttelmaschine geschüttelt, über Nacht stehengelassen und am nächsten Tag die Viskosität bestimmt.

$\mathrm{K}$ a m p f er. Es wurden Lösungen in reinem Azeton bestimmt und in Azeton, in dem 200 und $400 \mathrm{~g}$ Japankampfer pro Liter gelöst waren.

\begin{tabular}{|c|c|c|c|}
\hline \multirow[b]{2}{*}{ Versuch } & \multicolumn{3}{|c|}{ Lösungsmittel } \\
\hline & Azeton rein & $\begin{array}{c}20 \text { Proz. } \\
\text { Kampfer } \\
\text { Viskosita }\end{array}$ & $\begin{array}{l}40 \text { Proz. } \\
\text { Kampfer }\end{array}$ \\
\hline $\begin{array}{l}1 \\
2 \\
3 \\
4\end{array}$ & $\begin{array}{l}2 \cdot 36 " \prime \\
2^{\prime} 6^{\prime \prime} \\
1 \cdot 17 " \prime \\
3 \cdot 18 "\end{array}$ & $\begin{array}{l}4 \cdot 42 " \\
3 \cdot 29 " \\
1 \cdot 47 " \\
\text { unlöslich }\end{array}$ & $\begin{array}{c}6 \cdot 54 " \\
5,15 “ \\
2 \cdot 13 " \\
\text { unlöslich }\end{array}$ \\
\hline
\end{tabular}

Der Kampferzusatz hatte bei allen untersuchten Azetylzellulosen eirne Erhöhung der Viskosität zur Folge. Dies und besonders Versuch 4 scheint mir zu beweisen, dab die Löslichkeit der Azetylzellulosen in Azeton durch Kampferzusatz erniedrigt wird.

Triazetin. Als Lösungsmittel diente einerseits reines Azeton ${ }_{p}$ anderseits ein Azetontriazetingemisch mit 10 Vol.Proz. Triazetin. Die Azetylzellulosen lösten sich bei Zusatz von Triazetin viel leichter und schneller auf als in reinem Azeton.

\begin{tabular}{|c|c|c|}
\hline \multirow{3}{*}{ Versuch } & \multicolumn{2}{|c|}{ Lösungsmittel } \\
\hline & Azeton rein & 10 Proz. Triazetin \\
\hline & \multicolumn{2}{|c|}{ Viskositat } \\
\hline $\begin{array}{l}1 \\
2 \\
3 \\
4\end{array}$ & $\begin{array}{l}2 * 36^{\prime \prime} \\
2 \cdot 6 * \\
1 \cdot 17 " \\
3 \cdot 18^{\prime \prime}\end{array}$ & $\begin{array}{l}3 \cdot 23 " \\
3 \cdot 54 " \\
1 \cdot 59 " \\
4 \cdot 23 "\end{array}$ \\
\hline & & \\
\hline
\end{tabular}

Trotz der besseren Löslichkeit der Azetylzellulosen wird die Viskosität durch Triazetinzusatz erhöht. Die Exhöhung ist aber in diesem Fall wohl auf die ölige Beschaffenheit des Triazetins selbst zurückzuführen.

Dich lo r by drin. Als Lösungsmittel diente reines Azeton und Azetondichlorhydringemische mit 25 und 50 Vol.-Proz. Dichlorhydrin.

\begin{tabular}{|c|c|c|c|}
\hline & \multicolumn{3}{|c|}{ Lösungsmittel } \\
\hline Versuch & Azeton rein & $\begin{array}{c}25 \text { Proz. } \\
\text { Dichlorhydrin } \\
\text { Viskosität }\end{array}$ & $\begin{array}{c}50 \text { Proz. } \\
\text { Dichlorhydrin }\end{array}$ \\
\hline $\begin{array}{l}1 \\
2 \\
3\end{array}$ & $\begin{array}{l}2 \cdot 36 " \\
2^{\prime} 6^{\prime \prime \prime} \\
1 \cdot 17 "\end{array}$ & $\begin{array}{l}1.50 \text { " } \\
1 \cdot 51 " \\
1 \cdot 30 "\end{array}$ & $\begin{array}{l}3 \cdot 39 * \\
3 \cdot 15^{\prime \prime} \\
2,48 *\end{array}$ \\
\hline
\end{tabular}


Durch groben Dichorbydrinzusatz wird also die Viskosität immer erhöht. Bei der niederen Koncentration an Dichorhydin trit bei den einen Azelylzellulosen Erriedrigung (Versuch 1 und 2), bei den auderen Frhöhung (Versuch 3 und 4) der Viskositat ein. Dabei ist benetkenswert, dab auch Azerylzellulosen vom glechen Viskositatstypus sich verschieden vertialten.

p-Toluolsulfamid. Als Lösungsmitlel wurde reines $A z e t o n$ verwendet und Azeton mit $100 \mathrm{~g} \mathrm{p}$-Toluolsulfanid im Liter.

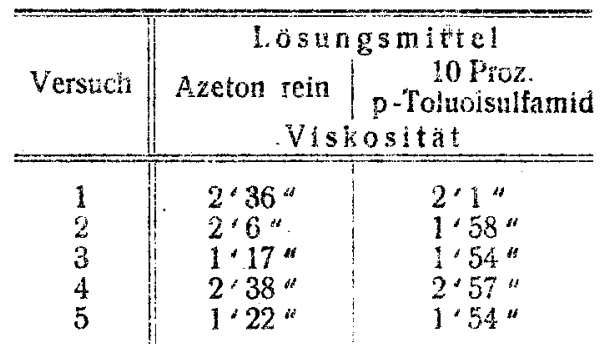

Auch dieser Zusatz wirkt also auf verschiedene Azetylzellulosen verschieden. Es gilt für die Beeinflussung der einzelnen Viskosiratstypen das oben für Dichlorhydrin gesagte. Zwischen dem Einflub von Dichlorhydrin und p-Toluo:sulfamid auf dic Viskositä der Azetyizeiluloselösungen scheint ein Parallelismss zu bestehen, was aus den Versuchen 1,2 und 3 ersichtlich ist.

Die Resultate mit Dichlorhydrin und p-Toluolsuliamid führen mich anf den Gedarken, ob es nicht möglich wäre, die Brauchbarkeit der verschiedenen Lösungsmittel und Zusätze für die Fabrikation zelluloidahnlicher Stoffe aus Azetylzellulosen durch solche Viskositätsmessungen in Laboratorium zu prüfen. Diesbezügliche Betriebsversuche konnten wegen Mangel an Material nicht ausgeführt werden.

\section{Zusammenfassung.}

1. Für die Beurteilung der Azetylzellulosen hinsichtlich ihrer Verwendbarkeit zur Herstellung plastischer Stoffe sind nicht die Viskositütszahien makgebend, sondern es sind Viskositätskurven erforderich.

2. Die untersuchten Ảzetylzellulosen lassen sich in sieben Viskositätstypen einteilen.

3. Ein Viskositätsminimum in Alkoholazetongemischen mit 90 Vol.-Proz. Azeton deutet stets auf unbrauchbare Azetylzellulosen.

4. Gute Azetylzellulosen weisen stets cin Viskositätsminimum in Alkoholazetonlösungen mit 80 Vol.-Proz. Azeton auf.
3. Zwishen dem guten Typ 1 und dem schlechen ryp 7 war irein Untershiad in der Viskositatskurye aufupinden.

6 Zusat won Japankampler und Triazetin erhobt die Vishosität der Azetylzciluloselosungen in reinem Azeton.

7. Zusatz von Dichlorhydrin und p.Toluolsulfamid wirkt auf die einen Azetyizellulosen viskositätserhöhend, auf die anderen viskositätsentiedrigend.

\section{An hang.}

Untersuchungen der Viskositäten von Nitrozelluloselosungen.

Da ich vorläufig nicht mehr Gelegenheit habe, auf dem Gebiete weiterzuarbeiten, gebe ich hier auch die Resultate meiner Nitrozelluloseuntersuchungen wieder.

Es wurden ein- bis dreiprozentige Nitrozelluloselösungen in Kampferalkohol untersucht. Als Lösungsmitte! diente Kampferalkohol mit 300, 400,500 und $750 \mathrm{~g}$ Japankampfer in einem Liter Alkohol. Die Lösungen wurden so hergestellt, daP in $100 \mathrm{ccm}$ des Lösungsmittels täglich je $1 \mathrm{~g}$ Nitrozeilulose eingeführt wurde, eine Arbeitsmethode, wie sie $\mathrm{Schwa}\left[z^{6}\right.$ ) in seiner Arbeit angegeben hat. Die Lösungen wurden sechs Stunden im Schüttelapparat geschütelt, über Nacht stehen gelassen und am folgenden Vormittag untersucht. Das O stwaldsche Visikosimeter, das für diesen $Z$ weck verwendet wurde, hatte folgende Dimensionen:

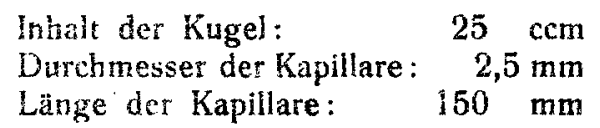

Die acht untersuchten Nitrozellulosen waren gebleicht, hergestellt aus Linters mit Schnittfaden, mit einem N-Gehalt, der zwischen 10,41 und 10,58 Proz. schwankte.

Bei den Viskosirätsmessungen ergaben sich für die Nitrozellulosen zwei Typen. Bei den einen sank die $V$ iskosităt mit steigendem Kamplergehait der Lösungen, z. B.:

\begin{tabular}{|c|c|c|c|}
\hline Kampfer- & \multicolumn{3}{|c|}{ Nitrozellulose } \\
\hline $\begin{array}{c}\text { konzentration } \\
\text { in Proz. }\end{array}$ & $\lg$ & $\begin{array}{c}2 \mathrm{~g} \\
V \text { iskosit }\end{array}$ & $3 \mathrm{~g}$ \\
\hline $\begin{array}{l}30 \\
40 \\
50 \\
75\end{array}$ & $\begin{array}{l}23,4 " \\
24,7 " \prime \\
26,8 “ \\
29,7 "\end{array}$ & $\begin{array}{l}1 \cdot 46,7 " \\
1 \cdot 38,8 " \\
1 \cdot 35,7 " \\
1 \cdot 34,0 "\end{array}$ & $\begin{array}{l}6 \cdot 0,2 " \\
5,32,5 " \\
5 \cdot 24,3 " \\
5 \cdot 18,7 “\end{array}$ \\
\hline
\end{tabular}

3) H.Schwarz, Koll-Zeitschr. 12, 32 (1913). 
Diese Art Nitrozellulosen erwies sich als sehr gut tür die Fabrikatior von Zelluloid. Das - Materiai war bei der Verarbeitung recht plastisch und zeigte auch in fertigem Zustand gute Resuitate.



Fig. 4

Der zweite Nitrozellulosetypus war dadurch charakterisiert, daB die Viskosität durch höheren Kampferzusatz erhöht wurde, z. B.

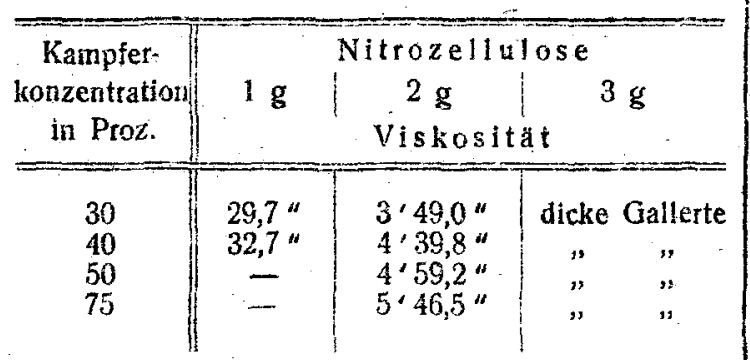

Nitrozellulosen von diesem Typus waren für die Pabrikation von Zelluloid fast unbrauchbar; sie lieferten ein "kurzes", zähes und trockenes Material. Das fertige Zelluloid war sehr spröde.

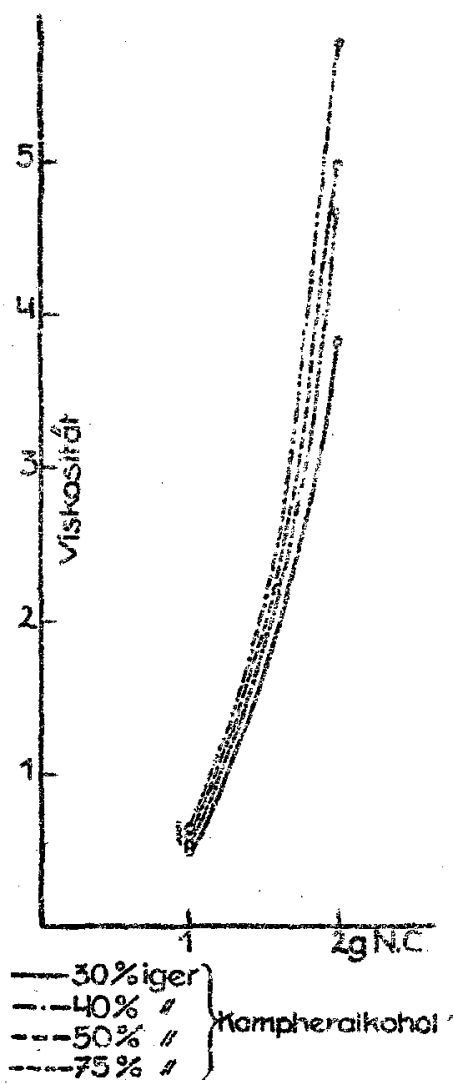

Fig. 5

Bei den acht untersuchten Nitrozellulosen zeigte sich stets, dab ein höherer Kampferzusatz bei guten Nitrozeliulosen eine Viskositätserniedrigung, bei schlechten eine Viskositätserhohung zur Folge hat. Auf diese Weise lassen sich wohl auch die entgegengesetzten Resultate von S chwarz ${ }^{7}$ ) und Nishidas) exklären. Die Verschiedenheit der Viskositätsbeeinflussung durch die Kampferkotzentration zeigt sich, jedoch erst in zweiprozentigen Nitrozelluloselosungen.

Herrn Dr. H. Schwarz, auf dessen Anregung die yorliegende Arbeit entstanden ist, möchte ich auch an dieser Stelle herzlichst danken für die talktäftige Unterstützung und das rege Interesse, das er dern Fortyang meiner Uriteruchungen sters entgegenbrachte.

3) H. Schwarz, Koll-Zeirscir. 12, 22 (1013),

5) H. Nislid a, Kunststoffe 4, 81 u. 105 (19:413) 